\title{
Transient idiopathic central diabetes insipidus: is severe sepsis a possible cause?
}

\author{
Ilaria Lazzari, ${ }^{1}$ Alessandro Graziani, ${ }^{2}$ Federica Mirici, ${ }^{2}$ Giuseppe Francesco Stefanini ${ }^{2}$ \\ ${ }^{1}$ Department of Internal Medicine, Sant'Orsola Hospital, Bologna; ${ }^{2}$ Department of Internal Medicine, Faenza Hospital, \\ Faenza (RA), Italy
}

\begin{abstract}
Idiopathic central diabetes insipidus (CDI) is a disorder characterized by hypotonic polyuria and polydipsia, without any identified etiology. Here we report a case of a 57-year-old woman, with idiopathic CDI, admitted to our department with severe sepsis and acute kidney failure. After clinical and radiological investigations, she was diagnosed with idiopathic CDI. In this case report the findings suggest that severe sepsis could be the trigger for this disease. In addition, we hypothesise that apelin, a diuretic neuropeptide, plays a role in such a process. Apelin levels are known to increase during severe sepsis, which in turn counteracts vasopressin actions through inhibition of vasopressin neuron activity and vasopressin release.
\end{abstract}

\section{Introduction}

Diabetes insipidus (DI) is a disease characterized by hypotonic polyuria, defined as a urine volume $>40$ $50 \mathrm{~mL} / \mathrm{kg}$ /day with urine osmolality $<300 \mathrm{mOsm} / \mathrm{kg}$, and consequent polydipsia. There are two forms of DI: central diabetes insipidus (CDI), due to a partial or total deficiency of antidiuretic hormone [arginine vasopressin (AVP) or vasopressin] and peripheral DI (PDI), where the $\mathrm{V} 2$ receptors in the kidney collecting tubule are resistant to the action of the hormone itself. Another condition, which shares similar clinical features, is known as psychogenic DI. However, the no-

Correspondence: Ilaria Lazzari, Department of Internal Medicine, Sant'Orsola Hospital, via Albertoni 15, 40138 Bologna, Italy.

E-mail: ilaria.lazzari17@gmail.com

Key words: Central diabetes insipidus; vasopressin; severe sepsis; apelin.

Acknowledgments: we thank Dr J. Cucchiarini for translation.

Conflict of interest: the authors declare no potential conflict of interest.

Received for publication: 26 March 2016.

Revision received: 22 June 2016.

Accepted for publication: 23 August 2016.

This work is licensed under a Creative Commons Attribution NonCommercial 4.0 License (CC BY-NC 4.0).

CCopyright I. Lazzari et al., 2017

Licensee PAGEPress, Italy

Italian Journal of Medicine 2017; 11:78-81

doi:10.4081/itjm.2017.717 table difference with this condition is that it does not involve vasopressin, but instead involves the presence of a psychiatric disorder called psychogenic polydipsia, which is characterized by an uncontrollable desire to drink fluid. ${ }^{1}$

In CDI, AVP deficiency may be caused by a defect in its production by the supraoptic and paraventricular nuclei of the hypothalamus or its secretion by cells of neurohypophysis. Causes of this disease can include genetic disorders, head trauma, tumors, vascular lesions, infections and systemic inflammatory diseases. ${ }^{2}$ Despite numerous etiologies, $30-50 \%$ of cases of CDI remain idiopathic. ${ }^{3}$

PDI can be the result of drug toxicity (lithium is one of the most common), obstruction of the urinary tract, acute tubular necrosis, metabolic disorders and genetic defects. $^{2}$

Here we describe a case of a patient who was admitted to our department with severe sepsis and acute kidney failure. After assessment, she was diagnosed with idiopathic CDI. This report suggests that severe sepsis may have been the trigger for the disease and examines the role of apelin in the mechanism underlying CDI.

\section{Case Report}

A 57-year-old woman was admitted to our department with acute renal failure, pyrexia and psychomotor retardation, which appeared a few days after performing a cystoscopy to study bladder prolapse. The patient had a history of hypothyroidism, chronic headache, hepatitis $C$ virus positivity and bipolar personality disorder on lithium treatment for more than 20 years. Lithium levels were slightly above the normal range $(1.74 \mathrm{mEq} / \mathrm{L})$ and for this reason the drug was immediately discontinued. 
On physical examination at the time of admission the patient's vital signs included a temperature of $38^{\circ} \mathrm{C}$, a heart rate of 104 beats $/ \mathrm{min}$, a respiratory rate of 16 breaths $/ \mathrm{min}$ and an altered mental state. Oxygen saturation was $95 \%$ without an oxygen supply. Blood tests showed the following values: white blood cells $11,660 / \mu \mathrm{L}(10,200 / \mu \mathrm{L}$ neutrophils $), \mathrm{C}$-reactive protein $150 \mathrm{mg} / \mathrm{dL}$, creatinine $3.51 \mathrm{mg} / \mathrm{dL}$, urea $79 \mathrm{mg} / \mathrm{dL}$, $\mathrm{Na}+129 \mathrm{mmol} / \mathrm{L}, \mathrm{k}+3.5 \mathrm{mmol} / \mathrm{L}$. Blood cultures and urine cultures were positive for Escherichia coli. The APACHE II (acute physiology and chronic health evaluation II) score was calculated at 24 , with a risk of mortality estimated at $49.7 \%$.

After initial investigations once the individual's renal function had sufficiently improved, the woman was subjected to an abdominal computed tomography with iodine contrast, which showed findings consistent with bilateral pyelonephritis and bilateral pleural effusions. The patient was subsequently diagnosed with severe sepsis from E. coli of urinary origin. She was treated with targeted antibiotics and adequate hydration, after which renal function was rapidly restored and pleural effusions regressed.

After approximately $96 \mathrm{~h}$, post-admission during the early resolution of the acute event, a progressive increase in urine output, up to a value of $12,000 \mathrm{~mL} /$ day was observed. Laboratory tests showed low levels of urine osmolality (up to $72 \mathrm{mOsm} / \mathrm{kg}$ ), high values of plasma osmolality (with peak of $304 \mathrm{mOsm} / \mathrm{kg}$ ) and hypernatremia (up to values of $155 \mathrm{mEq} / \mathrm{L}$ ). Upon examination, the patient appeared to be confused and exhibited the following conditions: drowsiness, ideomotor slowdown, low blood pressure with a compensatory rise in heart rate, arterial oxygen saturation to normal, intense xerostomia and a strong feeling of fatigue and muscle weakness. She also reported marked polydipsia, and by monitoring the daily intake of liquids taken by the patient, intake values up to $10,900 \mathrm{~mL}$ per day were recorded. Given the marked alteration of biochemical parameters and the risk of dehydration, it was decided not to conduct the fluid deprivation test. The patient was treated with low dose desmopressin $(5 \mathrm{mcg}$ spray per nostril twice daily) alongside serial blood and urine tests. The dosage was gradually increased to 120 mcg sublingual capsules twice a day after discharge from hospital, due to the beneficial effect it had which was evidenced by her improved clinical condition and laboratory data (Table 1).

Despite the patient's clinical history of lithium therapy and acute kidney failure, the response to desmopressin along with progressive reduction of diuresis and improvement of biochemical parameters, deposed for CDI diagnosis. To study the etiology of this disorder the patient underwent a magnetic resonance image (MRI) scan without contrast to study sella turcica. The scan showed little evidence of a dif-

Table 1. Laboratory values before and after the initiation of therapy with desmopressin.

\begin{tabular}{|c|c|c|c|c|c|c|c|}
\hline Day & $\begin{array}{c}\text { 24-h urine } \\
\text { volume (mL) }\end{array}$ & $\begin{array}{c}\text { Urine } \\
\text { osmolarity } \\
\text { (mOsm/kg) }\end{array}$ & $\begin{array}{c}\text { Plasma } \\
\text { osmolarity }\end{array}$ & $\begin{array}{l}\text { Plasma } \\
\text { sodium }\end{array}$ & $\begin{array}{c}\text { Plasma } \\
\text { creatinine } \\
(\mathrm{mg} / \mathrm{dL})\end{array}$ & GFR & $\begin{array}{l}\text { Incoming } \\
\text { liquids }\end{array}$ \\
\hline 1 & 2500 & - & - & 132 & 3.3 & 15 & - \\
\hline 3 & 3600 & - & - & 146 & 1.7 & 33 & - \\
\hline 4 & 2200 & - & - & 150 & 1.29 & 46 & - \\
\hline 5 & 5200 & - & - & 152 & 1.05 & 59 & - \\
\hline 6 & 7500 & - & - & - & 0.92 & 69 & 7500 \\
\hline 9 & 11,200 & 72 & 304 & 150 & 0.66 & 98 & 10,900 \\
\hline 13 & 12,000 & 99 & 294 & 145 & 0.55 & 100 & 7200 \\
\hline \multicolumn{8}{|c|}{ Begins desmopressin intranasal 2 mcg 2 times a day } \\
\hline 14 & 10,000 & 95 & 296 & 145 & - & - & 7700 \\
\hline 16 & 56,000 & - & - & 141 & - & - & 4200 \\
\hline
\end{tabular}

Begins desmopressin oral 60 mcg 2 times a day

\begin{tabular}{|c|c|c|c|c|c|c|c|}
\hline 19 & 3600 & - & 285 & 140 & - & - & - \\
\hline 27 & 3000 & - & - & 139 & - & - & - \\
\hline 1 month & 2200 & - & 290 & 142 & 0.79 & 89 & 2000 \\
\hline
\end{tabular}

After discharge begins desmopressin oral $120 \mathrm{mcg} 2$ times a day and stops it after three months

\begin{tabular}{lllllll}
\hline 4 months & 2000 & 380 & 292 & 142 & 0.76 & 91 \\
\hline
\end{tabular}

GFR, glomerular filtration rate. 
ference in signal intensity between the adenohypophysis and neurohypophysis. This finding alone, was not specific to any specific disease process, but instead suggested a posterior pituitary disorder. ${ }^{4}$ The secretory function of the hypophysis was then studied to assess the possible impairment of the gland. We assayed: follicle-stimulating hormone, luteinising hormone, oestradiol, growth hormone, prolactin, thyroid stimulating hormone, triiodothyronine, thyroxine, adrenocorticotropic hormone and cortisol. The basic hormonal assays were all within normal ranges. The patient was finally diagnosed with idiopathic CDI alongside severe sepsis of urinary origin.

In the months following discharge the patient demonstrated progressive clinical resolution alongside improved laboratory test values, reaching patient wellbeing with discontinuation of therapy after four months. A control MRI scan with contrast was completely negative.

\section{Discussion}

DI is a rare disease whose etiological diagnosis is often a complex challenge for the clinician. The monitoring of urine output and daily water intake, as well as the repeated determination of serum electrolytes, plasma osmolality and urinary osmolality are fundamental to facilitate diagnosis. The levels of plasma antidiuretic hormone (ADH) should be determined when available, but it is not considered a fundamental exam for the diagnosis of DI.

There is some clinical overlap between DI and psychogenic polydipsia, like the high flow of dilute urine and increased thirst. The distinctive element between DI and psychogenic polydipsia (in addition to response to the water deprivation test) is the presence of elevated serum sodium levels in the former compared to low serum sodium levels in the latter. ${ }^{5}$ In our case, the water deprivation test was not performed because the biochemical data were already sufficient for diagnosis, as well as the potential risks regarding severe dehydration and its related complications.

After confirming the presence of DI, the gradual response to the administration of desmopressin (DDAVP), a synthetic analogue of vasopressin, allowed the distinction between the central and nephrogenic form of the disease. The central form is linked to a lack of ADH and responds well to exogenous administration of the hormone usually with a gradual decrease in urine output and an increase in urinary osmolality, respectively. Conversely in nephrogenic DI, exogenous administration of ADH has no effect as the problem lies with the insensitivity of the renal collecting tubule to the hormone. ${ }^{6}$ In our case, after a few days of desmopressin therapy we noted a reduction in urinary output and an increase in urinary osmolality, which in turn led us to give a diagnosis of CDI. This excludes a nephrogenic form due to the lithium therapy (even though this drug was discontinued immediately upon admission, before the patient developed polyuria) or to the acute renal failure that she presented with when this came to our attention.

In order to understand the cause of CDI, a brain MRI without contrast was performed to study the sella turcica and to exclude an expansive process, signs of encephalitis or meningitis and vascular lesions. This radiological exam showed a normal-size pituitary gland without any focal parenchymal lesions, but it demonstrated a reduction of the normal hyper intense signal of neurohypophysis on T1-weighted sequences due to phospholipid or secretory granules contained within the pituicyte. ${ }^{7}$ Such radiological evidence in isolation does not have sufficient specificity in order to establish any etiologic diagnosis but does not exclude the diagnosis of CDI.

After reviewing many of the most common and known etiologies, we wondered whether the state of severe sepsis that occurred in the aforementioned patient could be responsible for the onset of neurogenic DI. There is a possibility that a systemic condition such as severe sepsis could determine the dysfunction of the hypothalamic-pituitary axis. In line with this, we assume that apelin can play a more or less decisive role in this hormonal disorder.

Apelin is a neuropeptide expressed in the supraoptic and paraventricular nuclei of the hypothalamus. It acts on localized vasopressinergic receptors in these neurons by inhibiting their activity and vasopressin release, and thus in turn acts as a diuretic. ${ }^{8,9}$ The apelinergic system is involved in the regulation of fluid homeostasis and it could be involved in pathogenesis of diabetes insipidus. ${ }^{10,11}$ Some case-control studies have shown that in patients with sepsis the levels of blood apelin are higher than in healthy subjects, and its increase is directly correlated with the severity of the condition of sepsis assessed by the APACHE II score. ${ }^{12,13}$

According to our assumption (which should also be supported by experimental studies), the reduction of vasopressin could be due, at least in part, to its own increasing levels of apelin during severe sepsis. We note that the patient in question began developing polyuria within four days of hospitalisation. These observations combined together with the answer to DDAVP and the MRI scan led us to come to the diagnosis of CDI during a septic state.

The normalisation of the clinical and radiological features after three months seems to confirm our hypothesis, deposing for a transitory form of CDI, regressed with complete resolution of the state of the septic patient. 


\section{Conclusions}

It is not currently known why only some patients could develop diabetes insipidus during a sepsis condition and other do not. It is likely that other factors support the role of apelin in the determination of CDI in severe sepsis and further investigations are necessary to identify this correlation. More data are certainly required to confirm our hypothesis that the state of severe sepsis is a possible cause of transitory CDI, but it could certainly explain many cases of idiopathic CDI.

\section{References}

1. Verbalis JG. Diabetes insipidus. Rev Endocr Metab Dis 2003;4:177-85.

2. Saifan C, Nasr R, Mehta S, et al. Diabetes insipidus: a challenging diagnosis with new drug therapies. Nephrol 2013;2013: 797620.

3. Maghnie M, Cosi G, Genovese E, et al. Central diabetes insipidus in children and young adults. $\mathrm{N}$ Engl $\mathrm{J}$ Med 2000;343:998-1007.

4. Di Iorgi N, Napoli F, Allegri AE, et al. Diabetes insipidus - diagnosis and management. Horm Res Pediatr 2012;77:69-84.

5. Dundas B, Harris M, Narasimhan M. Psychogenic poly- dipsia review: etiology, differential and treatment. Curr Psychiatry Rep 2007;9:236-41.

6. Fauci S, Braunald E, Kasper D, et al. Diabetes Insipidus. In: Harrison's principles of internal medicine, 17th ed. New York: Mc Graw Hill; 2008. pp 2145-2147.

7. Elster AD. Imaging of the sella: anatomy and pathology. Semin Ultrasound CT MR 1993;14:182-94.

8. De Mota N, Reaux-Le Goazigo A, El Messari S, et al. Apelin, a potent diuretic neuropeptide counteracting vasopressin actions through inhibition of vasopressin neuron activity and vasopressin release. Proc Natl Acad Sci U S A 2014; 11:10464-9.

9. O'Carroll AM, Lolait SJ, Harris LE, et al. The apelin receptor APJ: journey from an orphan to a multifaceted regulator of homeostasis. J Endocrinol 2013;219:13-35.

10. Ladeiras-Lopes R, Ferreira-Martins J, Leite-Moreira AF. The apelinergic system: the role played in human physiology and pathology and potential therapeutic applications. Arq Bras Cardiol 2008;90:343-49.

11. Urwyler SA, Timper K, Fenske W, et al. Apelin concentrations in patients with polyuria-polydipsia syndrome. J Clin Endocrinol Metab 2016;101:1917-23.

12. Xing B, Zeng Q. Relationship between the early level of serum Apelin of patients with sepsis and clinical prognosis. Shandong Med J 2009;49:26-8.

13. Wang Y, Zheng Y, Fu Y, Ding N. The changes and the clinical significance of plasma Apelin in elderly patients with sepsis. Chinese J Geriatr 2013;32:861-3. 